\title{
Diphenhydramine Hydrochloride/Dexamethasone/Nystatin Magic Mouthwash
}

National Cancer Institute

\section{Source}

National Cancer Institute. Diphenhydramine Hydrochloride/Dexamethasone/Nystatin

Magic Mouthwash. NCI Thesaurus. Code C82678.

An oral suspension containing diphenydramine hydrochloride, dexamethasone and nystatin, with anithistaminic, antiinflammatory, and antifung al activities. Diphenhydramine hydrochloride/dexamethasone/nystatin magic mouthwash inhibits the cytokine-mediated inflammation and yeast colonization of the oral mucosa associated with chemotherapy and radiation therapy. 